$4-1-2008$

\title{
Investigation of Magnetic Interactions in Large Arrays of Magnetic Nanowires
}

Petru S. Fodor

Cleveland State University, p.fodor@csuohio.edu

G. M. Tsoi

L. W. Wenger

Follow this and additional works at: https://engagedscholarship.csuohio.edu/sciphysics_facpub

Part of the Physics Commons

How does access to this work benefit you? Let us know!

Publisher's Statement

Copyright 2008 American Institute of Physics. This article may be downloaded for personal use only. Any other use requires prior permission of the author and the American Institute of Physics. The following article appeared in Journal of Applied Physics 103 (2008): $07 B 713$ and may be found at

\section{Original Citation}

Fodor, Petru S., G. M. Tsoi, and L. W. Wenger. "Investigation of Magnetic Interactions in Large Arrays of Magnetic Nanowires." Journal of Applied Physics 103 (2008): 07B713.

\section{Repository Citation}

Fodor, Petru S.; Tsoi, G. M.; and Wenger, L. W., "Investigation of Magnetic Interactions in Large Arrays of Magnetic Nanowires" (2008). Physics Faculty Publications. 8.

https://engagedscholarship.csuohio.edu/sciphysics_facpub/8

This Article is brought to you for free and open access by the Physics Department at EngagedScholarship@CSU. It has been accepted for inclusion in Physics Faculty Publications by an authorized administrator of EngagedScholarship@CSU. For more information, please contact library.es@csuohio.edu. 


\title{
Investigation of magnetic interactions in large arrays of magnetic nanowires
}

\author{
P. S. Fodor ${ }^{\mathrm{a})}$ \\ Physics Department, Cleveland State University, Cleveland, Ohio 44115, USA \\ G. M. Tsoi and L. E. Wenger \\ Department of Physics, University of Alabama at Birmingham, Birmingham, Alabama 35294, USA
}

(Presented on 7 November 2007; received 10 September 2007; accepted 29 October 2007; published online 25 January 2008)

\begin{abstract}
The magnetic interactions in large arrays of ordered magnetic nanowires with $12-48 \mathrm{~nm}$ diameter and 55-95 nm spacing were investigated using modified Henkel plots. The measurements for nanowire arrays ac demagnetized with the field applied parallel to the nanowire axis (the easy magnetization axis) indicate that the dominant interaction during the switching process is the magnetostatic coupling between the nanowires. Nevertheless, while the strength of the magnetostatic interactions increases with the magnetic moment associated with the nanowires, the increase is not linear with respect to the volume of the nanowires. Moreover, the dependence of the remanence curves on the field history suggests that even for magnetic nanowire systems with high geometric anisotropy, the magnetic pole structure of the nanowires can be complex. This conclusion is also supported by the field dependence of the initial magnetization curves. (C) 2008 American Institute of Physics. [DOI: 10.1063/1.2834242]
\end{abstract}

\section{INTRODUCTION}

The continuous demand for improved performance and increased areal density in magnetic storage applications has fueled a continually increasing interest in the fabrication and characterization of patterned arrays of magnetic nanostructures. In this context, patterned media, ${ }^{1}$ where each bit is physically separated from the others, is seen as a viable approach to address the randomization of the magnetization in nanoscale magnetic structures due to thermal effects. ${ }^{2}$

For the fabrication of patterned magnetic media, the main focus is developing synthesis techniques capable of producing features in the nanometer range while having the potential for cost effective mass production. Besides wellestablished methods such as e-beam lithography, ${ }^{3}$ a multitude of less conventional approaches that show promise have been investigated including deposition in nanotemplates, ${ }^{4}$ lithography assisted self-assembly, ${ }^{5}$ and nanoimprint lithography. ${ }^{6}$ On the other hand, the magnetic characterization research has concentrated on studying magnetic properties and magnetization switching mechanisms that emerge as the size of the magnetic particles becomes comparable with the exchange length and domain wall thickness. For example, magnetic force ${ }^{7}$ and Lorentz electron ${ }^{8}$ microscopies have shown that intricate domain structures such as magnetic vortices can be directly observed during the switching process. While understanding how the size and geometry affect properties such as coercivity and thermal stability is critical for the applicability of these systems for magnetic storage, it is equally important to understand the magnetic interactions in high density arrays and the dependence of these couplings on the magnetic structure of the individual nano-objects. ${ }^{9,10}$

In this paper, we investigate the magnetic interactions in

${ }^{a)}$ Electronic mail: p.fodor@csuohio.edu ordered arrays of electrodeposited magnetic nanowires. For all the arrays investigated, the magnetostatic coupling between the nanowires plays an important role during the switching process as expected for a system of closely packed magnetic particles with large geometric anisotropy. Nevertheless, the strength of the magnetostatic coupling between the nanowires is dependent on the magnetic field history. This indicates that despite the large aspect ratio of the nanowires, multidomain magnetic structures can be induced within the individual nanowires.

\section{EXPERIMENTAL DETAILS}

Details on the fabrication of the nanowire arrays are presented elsewhere. ${ }^{11}$ In brief, $\mathrm{Co}_{0.45} \mathrm{Fe}_{0.55}$ alloy nanowires are electrodeposited in porous hexagonally ordered anodic alumina templates prepared using a multistep anodization process. The spacing between the nanowires was $D_{i}=55$ or $95 \mathrm{~nm}$, depending on the templates used. The diameter of the nanowires ranges from 12 to $48 \mathrm{~nm}$, while their length is $12 \mu \mathrm{m}$. The choice of the $\mathrm{Co}_{0.45} \mathrm{Fe}_{0.55}$ alloy was motivated by its nearly zero magnetocrystalline anisotropy, ${ }^{11}$ insuring that the properties of individual nanowires are dominated by shape anisotropy.

The geometry of the prepared templates was characterized using scanning electron microscopy (Hitachi model S-2400) while the magnetic measurements were performed with a super conducting quantum interference device magnetometer (Quantum Design model MPMS-5S). The dominant interactions in this magnetic system were investigated by analyzing two different remanence curves, i.e., the isothermal remanent magnetization $M_{r}(H)$ and the de demagnetization remanence $M_{d}(H)$. To measure the de demagnetization remanence $M_{d}(H)$, the sample is saturated in a high field. The field is then removed and $M_{d}(0)$ is measured. A small 


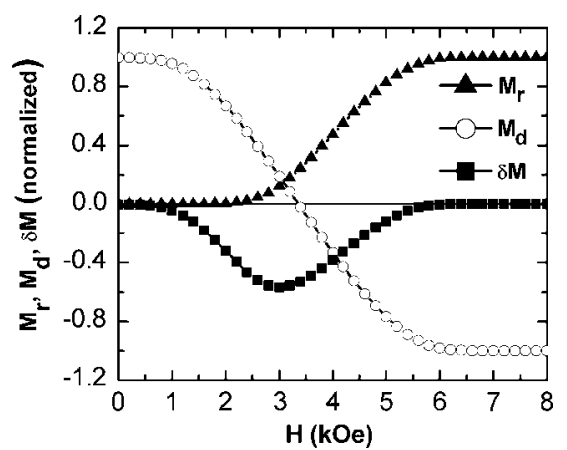

FIG. 1. Normalized isothermal remanent magnetization $M_{r}(H)$, dc demagnetization remanence $M_{d}(H)$, and modified Henkel $\delta M$ plots for $\mathrm{Co}_{0.45} \mathrm{Fe}_{0.55}$ alloy nanowires at room temperature $\left(D_{i}=12 \mathrm{~nm}, D_{p}=55 \mathrm{~nm}\right)$. All experimental data are normalized to the $M_{r}(8 \mathrm{kOe})$ value.

reversed field is subsequently applied and then removed, and the magnetization $M_{d}(H)$ is measured again. This process is repeated until the reversed field is equal in magnitude to the initial field at which the sample was saturated. This measured magnetization curve reflects only the irreversible changes in the sample since the measurements are always performed at zero field. The isothermal remanent magnetization $M_{r}(H)$ is measured in a similar way but the measurement starts with the sample in an ac demagnetized state. The two sets of magnetization data are then used to compute

$$
\delta M=\frac{M_{d}(H)}{M_{r}(\infty)}-\left[1-2 \frac{M_{r}(H)}{M_{r}(\infty)}\right],
$$

where the values for $M_{d}$ and $M_{r}$ are normalized with respect to the isothermal remanence for high fields. Thus $\delta M$ is effectively two times the difference between the fractions of particles switched by the applied field in the two different modes. Typical remanence and $\delta M$ (also known as modified Henkel of Kelly Henkel plot $^{12}$ ) curves versus the magnetic field $H$ are shown in Fig. 1.

\section{RESULTS AND DISCUSSION}

Figure 2 shows typical room temperature $\delta M$ plots for the $\mathrm{Co}_{0.45} \mathrm{Fe}_{0.55}$ nanowire arrays demagnetized with the field applied parallel to the long axis of the nanowires (their easy magnetization axis). Similar curves have been obtained for other compositions, temperatures, and even for pure element nanowires, making our results quite general for electrodeposited magnetic nanowire arrays. For all samples, the $\delta M$ curves exhibit negative peaks centered close to the coercivity. In the modified Henkel plot formalism, negative $\delta M$ values indicate that the magnetization reversal process is dominated by magnetostatic interactions. For our arrays where the geometric anisotropy of each nanowire is very large, this conclusion can be qualitatively explained considering each nanowire as a single domain with only two possible states, parallel (up) and antiparallel (down) to the nanowire axis. For a system of such interacting nanowires, the magnetostatic interactions are minimized for an antiparallel alignment of neighboring nanowires. Consequently, in the zero magnetization state, the stray fields from the neighboring nanowires essentially increases the external field required to
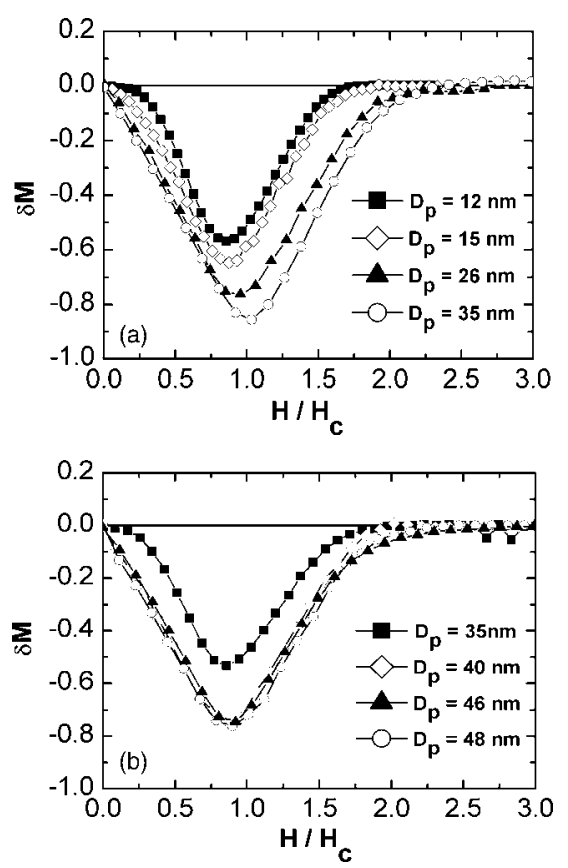

FIG. 2. Modified Henkel plots for $\mathrm{Co}_{0.45} \mathrm{Fe}_{0.55}$ alloy nanowire arrays with diameter ranging from (a) 12 to $35 \mathrm{~nm}$ at an interpore distance of $55 \mathrm{~nm}$ and (b) 35 to $48 \mathrm{~nm}$ at an interpore distance of $95 \mathrm{~nm}$. The zero magnetization state is obtained by ac demagnetization with the field applied parallel to the nanowire axis.

switch the magnetization of a single nanowire, while when starting from the saturated magnetization state, the magnetostatic coupling has the opposite effect. This leads to a lag between the applied field, at which the reversal process starts in the isothermal remanent magnetization, when compared with the dc demagnetization remanence, giving $\delta M$ negative values.

The $\delta M$ plots provide direct experimental evidence that the magnetic nanowires arrays demagnetized with the field parallel to the nanowire axis are coupled through interactions that are magnetostatic in nature. This conclusion is consistent with reports in similar systems in which the magnetostatic coupling effects were inferred indirectly from the analysis of the hysteresis loops. ${ }^{10,13,14}$ Moreover, in our study, we observe a more consistent dependence of the strength of the magnetostatic coupling on the diameter of the nanowires than in earlier reported data, presumably due to the lack of inhomogeneous magnetization states for this particular magnetic field configuration and history. ${ }^{4}$ However, as seen in the experimental data, while the amplitude of the peaks in the modified Henkel plots increases as the nanowire diameter is increased, this change does not scale linearly with the magnetic moment associated with the nanowires, i.e., proportional to the volume of the nanowires, as expected in a simple dipole approximation. A quantitative description of the magnetostatic coupling would require considering the multipolar components of the stray fields, especially when the nanowires are in very close proximity. ${ }^{15}$

On the other hand, while the above experimental data can be explained by considering the arrays as formed of single domain interacting nanowires, measurements in which the zero magnetization state is produced by dc demagnetiza- 


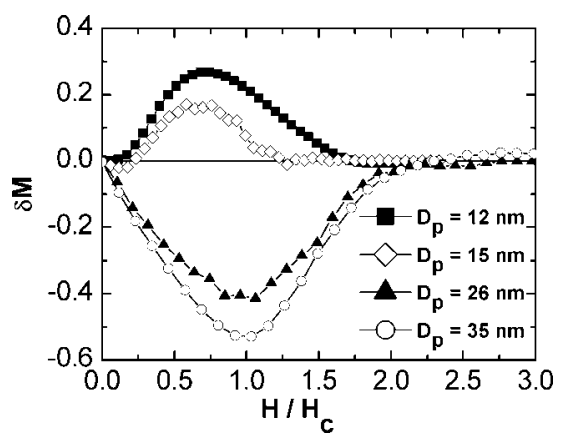

FIG. 3. Modified Henkel plots for $\mathrm{Co}_{0.45} \mathrm{Fe}_{0.55}$ alloy nanowire arrays with diameter ranging from 12 to $35 \mathrm{~nm}$ at an interpore distance of $55 \mathrm{~nm}$. The samples were demagnetized with fields applied perpendicular to the nanowire axis.

tion in a field perpendicular to the nanowire axis, show a more complicated magnetic behavior. While the $M_{d}(H)$ plots are identical to those described previously, for the 12 and $15 \mathrm{~nm}$ samples the $\delta M$ curves (Fig. 3) display positive peaks centered at field values smaller than the coercivity. As the diameter of the nanowires increases, the amplitude of this peak gets smaller and shifts to values closer to the coercivity. For the largest diameters, the modified Henkel plot peaks are negative, similar to the samples ac demagnetized with the field parallel to the nanowire axis, but the amplitude of the negative peak is reduced. Thus, in particular, for the small diameter nanowires, the magnetization switching is not dominated by the magnetostatic interactions that favor their antiparallel alignment. Typically positive $\delta M$ peaks are associated with exchange interactions which favor the parallel alignment of the magnetization, having an opposite effect to the magnetostatic ones. In the system studied, the only exchange interactions are within the nanowires since the distance between the nanowires and the insulating matrix separating them precludes any exchange interaction between the nanowires. This change in the strength of the exchange interactions can only be related with the presence within the nanowires of magnetization states different from the single domain ones favored by the shape anisotropy. The presence of multiple domain states within single nanowires is also apparent in the initial magnetization curves (Fig. 4) which are dependent on the initial demagnetized state. For samples demagnetized with the field parallel to the nanowire axis, no changes in the magnetization is observed until the applied

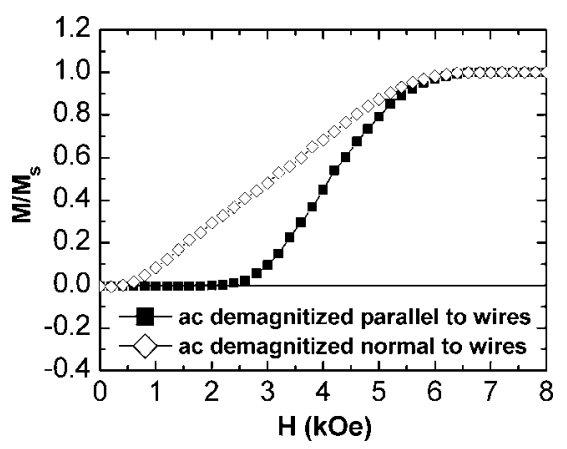

FIG. 4. Initial magnetization curves for nanowire arrays with $D_{p}=15 \mathrm{~nm}$, obtained from different initial magnetization states. field is close to the coercivity, i.e., it is strong enough to initiate magnetization reversal. In comparison, large changes in magnetization occur even for small magnetic fields for nanowire arrays demagnetized perpendicular to their main axis, since in the case of multidomain states there are no energy barriers associated with localized nucleation of magnetization reversal. The existence of multidomain states is supported by magnetic force microscopy studies on individual electrodeposited magnetic nanowires of similar diameters, which showed that the remanent state remaining after a saturation field is applied perpendicular to the nanowire axis consists of axial domains separated by $180^{\circ}$ walls. ${ }^{16}$ Recently, it has been argued that the presence of domain walls can also contribute to positive values in the $\delta M$ plots. ${ }^{17}$

In summary, magnetization measurements on magnetic nanowires embedded in anodic alumina templates have been used to analyze the nature of their magnetic interactions. The experimental data show that the magnetic nanowires studied cannot be treated as noninteracting systems but rather as strongly coupled through magnetostatic interactions. While qualitatively this interactions can be understood by treating the nanowires as dipoles, a quantitative description requires the consideration of multipolar contributions to the interaction field. Moreover, despite the measurements being performed on assemblies with large numbers of nanowires, their field history dependence gives insights in the switching process in individual nanowires.

\section{ACKNOWLEDGMENTS}

This work is supported in part by the National Science Foundation through Grant No. DGE-9870720.

${ }^{1}$ B. D. Terris and T. Thomson, J. Phys. D 38, R199 (2005).

${ }^{2}$ A. Moser, K. Takano, D. T. Margulies, M. Albrecht, Y. Sonobe, Y. Ikeda, S. H. Sun, and E. E. Fullerton, J. Phys. D 35, R157 (2002).

${ }^{3}$ S. Y. Chou, IEEE Trans. Magn. 85, 652 (1997).

${ }^{4}$ M. Sung, G. Zangari, M. Shamsuzzoha, and R. M. Metzger, Appl. Phys. Lett. 78, 2964 (2001).

${ }^{5}$ M. Farhoud, J. Ferrera, A. J. Lochtefeld, T. E. Murphy, M. L. Schattenburg, J. Carter, C. A. Ross, and H. I. Smith, J. Vac. Sci. Technol. B 17, 3182 (1999).

${ }^{6}$ S. Y. Chou, P. R. Krauss, and P. J. Renstrom, J. Vac. Sci. Technol. B 14, 4129 (1996).

${ }^{7}$ S. Goolaup and A. O. Adeyeye, Phys. Rev. B 73, 104444 (2006).

${ }^{8}$ P. Vavassori, N. Zaluzec, V. Metlushko, V. Novosad, B. Ilic, and M. Grimsditch, Phys. Rev. B 69, 214404 (2004).

${ }^{9}$ S. H. Liou, M. Zheng, M. L. Yan, R. Skomski, N. I. Polushkin, and D. J. Sellmyer, Scr. Mater. 44, 1347 (2001).

${ }^{10}$ K. Nielsch, R. Hertel, R. B. Wehrspohn, J. Barthel, J. Kirschner, U. Gösele, S. F. Fischer, and H. Kronmüller, IEEE Trans. Magn. 38, 2571 (2002).

${ }^{11}$ P. S. Fodor, G. M. Tsoi, and L. E. Wenger, J. Appl. Phys. 91, 8186 (2002).

${ }^{12}$ P. E. Kelly, K. O'Grady, P. I. Mayo, and R. W. Chantrell, IEEE Trans. Magn. 25, 3881 (1989).

${ }^{13}$ M. Ciureanu, F. Beron, L. Clime, P. Ciureanu, A. Yelon, T. A. Ovari, R. W. Cochrane, F. Normandin, and T. Veres, Electrochim. Acta 50, 4487 (2005).

${ }^{14}$ M. Vázquez, K. Nielsch, P. Vargas, J. Velázquez, D. Navas, K. Pirota, M. Hernández-Vélez, E. Vogel, J. Cartes, R. B. Wehrspohn, and U. Gösele, Physica B 343, 395 (2004).

${ }^{15}$ J. Velázquez, K. R. Pirota, and M. Vázquez, IEEE Trans. Magn. 39, 3049 (2003).

${ }^{16}$ Y. Henry, K. Ounadjela, L. Piraux, S. Dubois, J. M. George, and J. L. Duvail, Eur. Phys. J. B 20, 35 (2001).

${ }^{17}$ S. H. Liou, M. Zheng, M. L. Yan, R. Skomski, N. I. Polushkin, and D. J. Sellmyer, Scr. Mater. 44, 1347 (2001). 